\title{
Novel Compact Waveguide Optical Chemical Sensor for Control of Gaseous Ammonia Concentration
}

\author{
Nikolae V. Masalsky \\ Research Institute of System Researches RAS, Nakhimovskii pr. 36, korp. 1, Moscow, 117218, Russia \\ *Corresponding Author: volkov@niisi.ras.ru
}

Copyright (C) 2013 Horizon Research Publishing All rights reserved.

\begin{abstract}
The operation principle and construction of the waveguide optical chemical sensor control of absorbing type is considered. Characteristics of a sensor element on the basis of the waveguide structure generated on a Y-cutoff of lithium niobate for control of level of gaseous substances, in particular ammonia are experimentally researched. Potential possibilities of data application of instruments in different industries, ecology and telecommunication technologies are considered.
\end{abstract}

Keywords An Absorbing Chemical Sensor, The Waveguide Optical Structure, Ammonia, Lithium Niobate

\section{Introduction}

The detection of dangerous and harmful gases represents an important issue in the modern society. Ammonia $\left(\mathrm{NH}_{3}\right)$, as it is known, along with a nitrogen dioxide, nitrogen oxide, carbon oxide and a sulfur dioxide enters, in the list of the most harmful substances. Apart from its natural origin, it has many sources. The decision of this problem has priority value practically in all vital branches for the person, including the chemical, metallurgical, oil and gas industry, ecology, medicine, microelectronic, and defense [1-3]. The control of atmospheric ammonia levels is especially important because it is a flammable and explosive gas.

In recent years, various types of optical chemical sensors have been actively developed, which is due to a number of their advantages - high sensitivity, fast response, simplicity of signal multiplexing, possibility of distant measurement, small mass and overall dimensions and application of integrated technologies [2-5]. Among those available, waveguide optical chemical sensors are the most promising $[3,5,6]$. Their operating principle is based on the variation in the transmission of certain property-dependent laser wavelengths by the gas being monitored. If gaseous (gas, vapor) with a characteristic absorption line coinciding with the laser radiation wavelength emerges in the air at a waveguide close to the sensor, one observes an attenuation of the guided-mode power, since the mode field is damping exponentially in the air [3-5]. This effect is the basic for operation of absorption waveguide integrated optical chemical sensors.

The main challenge is to study and develop an easy-to-use, compact, reliable, and accurate waveguide integrated optical chemical sensors for discovering various ammonia gaseous substances in the atmosphere, including those extremely dangerous in ultra small concentrations.

\section{Waveguide Optical Chemical Sensor}

The functional scheme of the waveguide optical chemical sensor (WOCS) is shown on Figure 1.

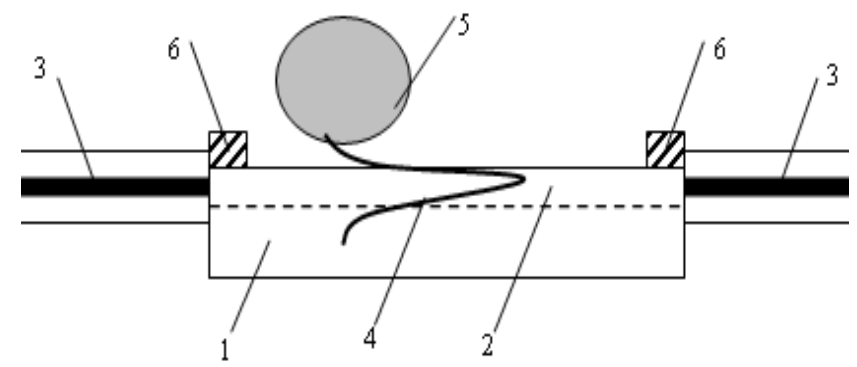

Figure 1 Schematic of the waveguide sensor: (1) substrate, (2) waveguide structure, (3) optical fiber, (4) optical field distribution, (5) area interaction, (6) optical power absorber

The $x$ axis is directed along the propagation of the optical beam( along optical axis), the $y$ axis is orthogonal to the waveguide plane and directed to its depth, and the $z$ coordinate is perpendicular to the direction of propagation of the optical beam.

The coherent optical radiation from the laser by means of an optical fiber (3), is directed into the waveguide structure (2), propagation at it, partially penetrating into air over a waveguide, and in the presence of a researched gas component on an output there is a reduction of the signal power, registered by the photo receiver element which is connected with WOCS by means of an optical fiber (3). 
Distinctive characteristics of the discussed unit in comparison with exiting analogues [2-4] are planarity, simplicity of manufactory, absence of a difficult adjustment, possibility of remote measurement. The waveguide structure is fabricated on a substrate (1) from optically transparent material, in our case, lithium niobate. Lithium niobate was selected as substrate material because of technology experience for this material in integrated optics and its good quality for optical waveguide manufacturing $[5,7,8]$. The waveguide structure is the main element of the WOCS. We mainly used localized $T E$ mode. The field intensity of the waveguide mode in the waveguide layer is distributed gaussally, and in the coating layer (air) and substrate, exponentially with distance from the waveguide layer [8]. The choice of the waveguide type and the method of laser-radiation input/output is determined by the sensor design, type of substances under study, and requirements of the integrated optical sensors.

The waveguide structure consists of two channel and two horn waveguides (see Figure 2). The WOCS effective length is approximately equal to the doubled length of the horn waveguide.

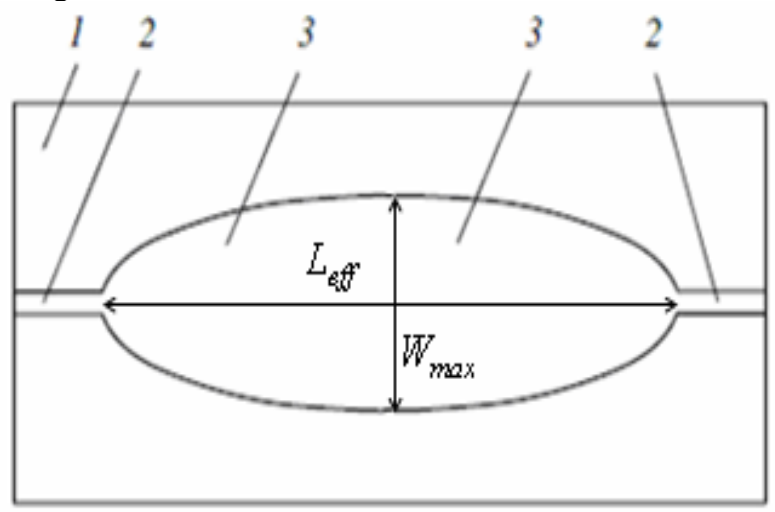

Figure 2. The waveguide structure topology: (1) substrate, (2) channel optical waveguide, (3) horn optical waveguide

The waveguide structure topology is projected so that, at first, it supported only since $T E$ polarization waveguide mode and, secondly, to provide minimum optical losses. The waveguide structure is fabricated by solid-state thermo diffusion of the titan into a Y-cut lithium niobate. The technology of its formation explicitly is considered in $[5,8]$. For titanium diffused waveguides the theoretical and experimental research leads us to a profile function for the refractive index normal to the boundary surface of the waveguide given by [5]:

$$
n^{2}(y)=n_{s}^{2}+\left(n_{0}^{2}-n_{s}^{2}\right)\left[(1-\alpha) e^{-\left(\frac{y}{h_{D}}\right)^{2}}+\alpha e^{-\frac{y}{h_{D^{\prime}}}}\right]
$$

where $n_{s}$ is the substrate refractive index, $n_{0}$ is the maximum the refractive index related to Ti-diffusion near the surface, $h_{D}$ is the effective depth of the Ti-profile, $h_{D^{\prime}}$ is the depth of the for out-diffusion, $\alpha$ is the fraction of the exponential profile and shown on Figure 3.

This result was achieved by the optimization of the profile function comparing calculated and measured values of the effective refractive indices for multimode waveguides [5].

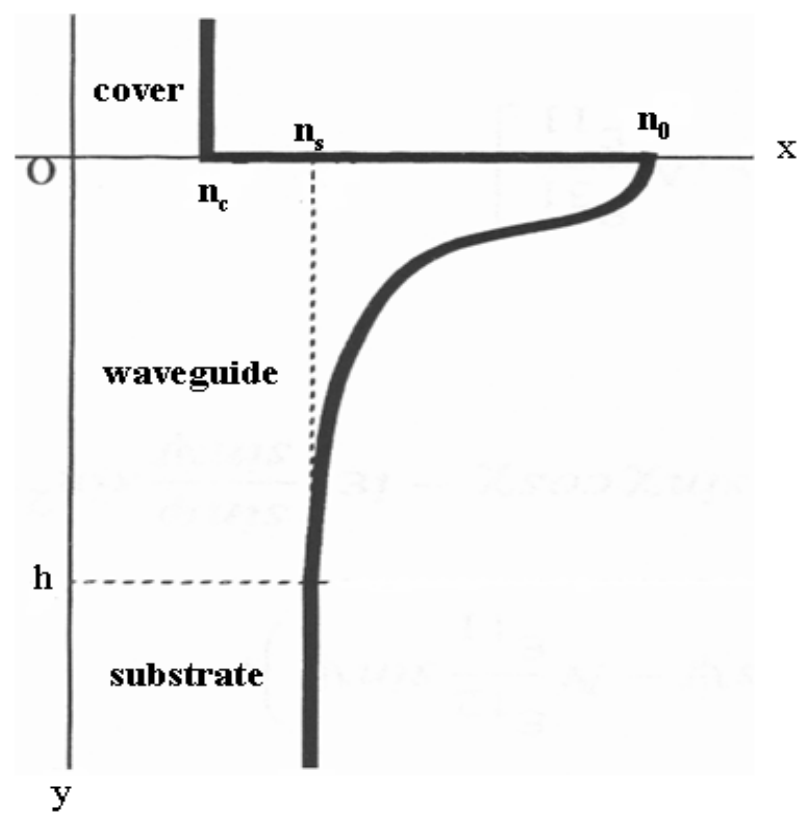

Figure 3. Refractive index distribution

Optical losses are an important parameter affecting the sensor designing. There are a lot of mechanisms for optical losses in this device. There are losses in the connection system for the fiber and the channel waveguide, as well as from Fresnel reflection and bulk and surface optical scattering in the waveguides $[5,7]$. In this case, Fresnel reflection and optical scattering in the waveguides depend on their optical lengths. The total optical transmission coefficient is

$$
K_{t o t}=K_{f-c h}^{2} K_{h}^{2} 10^{-q},
$$

where $K_{f-c h}$ is the optical transmission coefficient for the connection system of the fiber and the channel waveguide, $K_{h}$ is the optical transmission coefficient for the horn waveguide, and $q$ is the quantity that corresponds to optical losses due to irregularities and Fresnel reflection.

For optical propagation analysis it is necessary to calculate the diffraction integral. In first approximation we suppose that the optical beam aperture function in the planar waveguide is a Gauss function.

The optical beam is input into the channel waveguide from the fiber by means of end-fire coupling [5, 8]. High precision connection and the high quality of the fiber and crystal edges are needed for this method. The optical transmission coefficient in this case is defined by the overlap integral of fiber and channel waveguides' optical fields. Method of choice of the channel waveguide parameters is discussed in $[5,8]$. For such waveguides the relation exists between topology parameters $\left(w_{c h}\right.$ - width, $h_{c h}$ - depth), refractive index distribution and waveguides 
characteristics ( $\mathrm{m}$ is the waveguide mode's number, $\mathrm{N}$ is the effective refractive index) [7]. We use the effective refractive index method [9] to simulate channel and horn waveguide parameters. The purpose of this calculation is to find waveguide parameters that will decrease losses in passed optical radiation in a connection system of a fiber and a channel waveguide. With good accuracy zero mode optical fields both in fibers and waveguides are described by Gaussian function. Transmitting coefficient $K_{f-c h}$ becomes rather big (close to 1 ) if the value of radius $r_{0}$ of fiber mode correspond condition [5]. $4 r_{0}^{2}=4 a \lambda_{0} /\left(\pi \sqrt{\left.n_{1} \Delta n\right)}=w_{y} w_{z}\right.$ and ratio $w_{y} / w_{z}$ is approximately equal to 1 . Here the following denotes are used: a is a fiber inner core radius, $\lambda_{0}$ is wavelength in vacuum $\mathrm{n}_{1}$ is a fiber refractive index, $\Delta n$ is the difference of refractive indices for optical fiber in the central core and in the cladding, $w_{y}, w_{z}$ is the channel waveguide effective field width for level $1 / e$ in $\mathrm{y}$ and $\mathrm{z}$ directions. Linear displacement errors under fiber and channel waveguides coupling reduces transmission coefficient. It is necessary for achieving $K_{f-c h}=89 \%$ to limit these errors by $\Delta_{(y, z)}<0.1 r_{0}$, and for $K_{f-c h}=50 \%-$ by $\Delta_{(y, z)}<0.8 r_{0}$. The gap between the fiber and waveguide edges must be less than $\Delta_{x}<\pi n_{i m} w_{c h}^{2} / 4 \lambda_{0}$ in order to have a satisfactory transmission coefficient $\left(\mathrm{n}_{\mathrm{im}}\right.$ is refractive index of material placed between coupling components) [7, 8].

Research of the lithium niobate Ti-diffused optical horn waveguides shows that the horn waveguide optical width must grow more slowly than the increase in the optical width due to diffraction in order for the apparatus to have low optical losses [5]. According to the optimal horn, the low contour is

$$
W_{h}(x)=\left(w_{c h}^{2}+\frac{2 \lambda_{0} B}{N_{e f f}(x)} x\right)^{1 / 2}
$$

level, $N_{\text {eff }}(x)$ is the effective refractive index of the horn waveguide, and $w_{c h}$ is the channel waveguide width. We assume that losses due to the horn are small, because the horn contour has been selected with $B<1$. In this case, the horn transmission coefficient $K_{h}$ is more than $96 \%$ when $B=$ $0.91[7]$.

With specially selected thin films or immersion liquids one can reduce the Fresnel reflection down to $0.2 \mathrm{~dB}$.

Optical waveguides do not have ideal structures. There are irregularities in the waveguide bulk and on the waveguide boundaries [5, 7]. These irregularities produce optical scattering and, consequently, result in optical losses. The bulk optical irregularities are small, and the value for boundary deviations from an ideal plane is $0.05 \mu \mathrm{m}$ [7].

\section{Experimental Investigation}

On Figure 4 schematic of the experimental measurement setup for research of characteristics of the dissociated WOCS is shown. As a source of the coherent radiation the helium-neon laser with the wavelength $628.3 \mathrm{~nm}$ coinciding with absorption band of ammonia was used. The following absorption bands of gaseous ammonia are known in classical (nonlaser) spectroscopy: 110, $230 \mathrm{~nm}$ and near 790, 1300, 3000, 9000, 10300 and $10700 \mathrm{~nm}[1,2]$. A less studied $\mathrm{NH}_{3}$ absorption region is in the range of 549-653 $\mathrm{nm}[3,6]$. At the same time, particular features of operating various integrated optical sensors in the visible range have been investigated insufficiently, too. Selectivity of the offered approach is defined by laser radiation stability. For the experimental investigation we carried out preliminary rejection of the lasers. As photo receiving element photodiodes FD-303 have been used. The main waveguide part of WOCS was manufactured on Y-cut lithium niobate substrate with dimensions $48 \times 7 \times 1.5 \mathrm{~mm}$. The waveguide structure had following parameters: $L_{e f f}=42 \mathrm{~mm}, W_{\max }=158 \mu \mathrm{m}$. Primary benefits of the researched sensor in comparison with existing analogues [2-4] are insensitivity to external electromagnetic radiations and high interference free feature from collateral atmospheric phenomena.

where $B$ is the constant factor connected to the optical loss

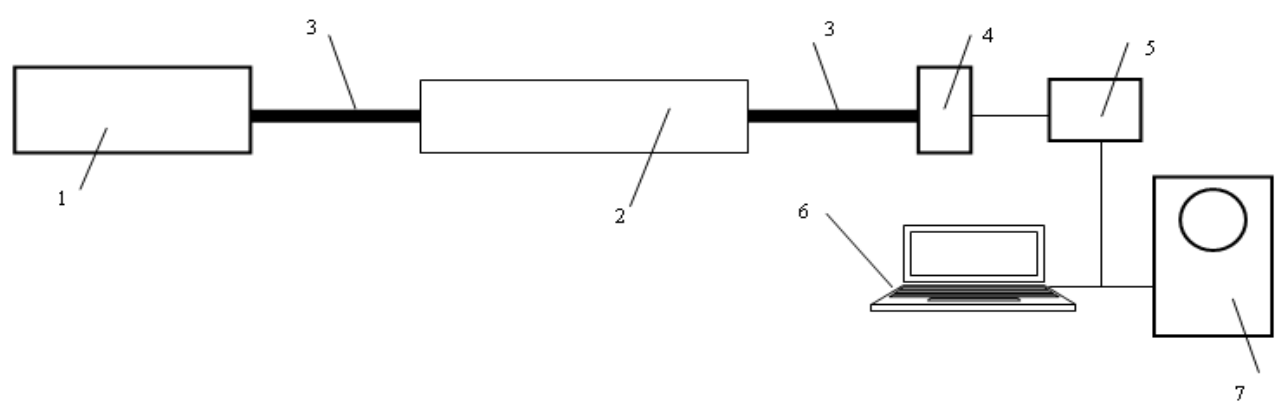

Figure 4. Schematic of the experimental measuring setting, where 1 - the laser, 2 - WOCS, 3 - an optical fiber, 4 - the photoreceiver element, 5 - the electronic block, 6 - the computer, 7 - an oscillograph large figure in one column 
The incident optical beam from the laser is directed into the input single mode optical fiber facet connecting to laser. The radiation emerging from the output optical fiber is registered by a photodetector, whose signal is fed to an evaluation electronic block. It consists of precision amplifiers with small displacement voltages and high voltage amplification factors. After post processing the signal is arrived on an oscillograph for a visual rapid analysis in real time and the computer where it was compared to beforehand written down reference signal without ammonia presence. The using of signals from the reference and sensor photoreceivers makes it possible to increase the signal-to-noise ratio and improve the sensitivity of the sensor. The subsequent mathematical handling of experimental data expands metrological possibilities

At testing the experimental sample has been placed in a hermetic container in which air with various concentration of gaseous ammonia in a range from $100 \mathrm{mg} / \mathrm{m}^{3}$ to $2 \mathrm{mg} / \mathrm{m}^{3}$ arrived at identical pressure (750 Torr), temperature $220 \mathrm{C}$ and damps of $75 \%$. Note, that the attenuation of optical power at the output of the sensor in the absence of ammonia is $8.4 \mathrm{~dB}$. After the next measurement the researched air compound completely was deleted that was supervised on an oscillograph and the computer. Before an admission of a new researched compound the reference signal registered. The results of measurements represented on Figure 5 show that dependence of average amplitude of the sensor response control almost linearly depends on ammonia level in a gas compound, if to provide its value in logarithmic scale. Then the range of change of data is transformed from 2 to 0.3 conditional units.

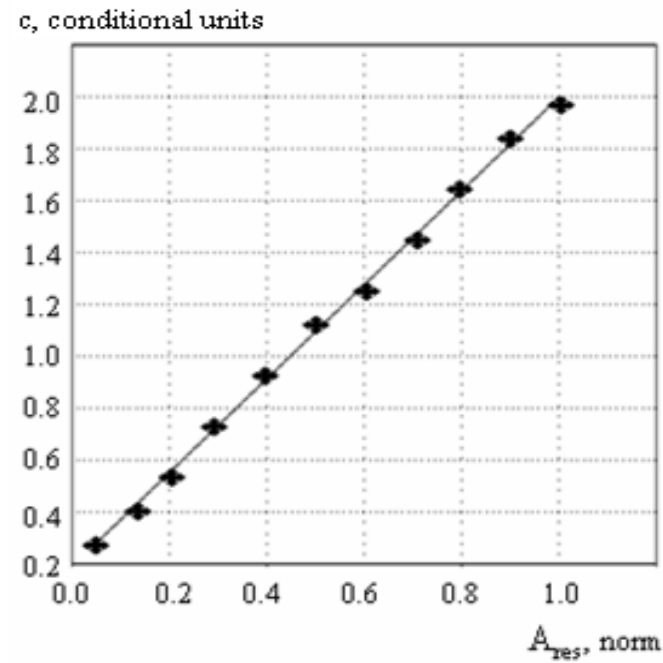

Figure 5. Dependence of ammonia concentration from the normalized amplitude of the response. By tags the experimental points are shown

It is note that the researched range of ammonia concentration exceeds maximum permissible concentration (maximum concentration limit) of the given substance (for the Russian Federation) in air of the occupied places $(0.2$ $\mathrm{mg} / \mathrm{m}^{3}$ ). Thus maximum concentration limit for an industrial zone $\left(20 \mathrm{mg} / \mathrm{m}^{3}\right)$ gets to a researched range.

On Figure 6 results of the experimental response of the device on change of ammonia concentration in air are resulted.

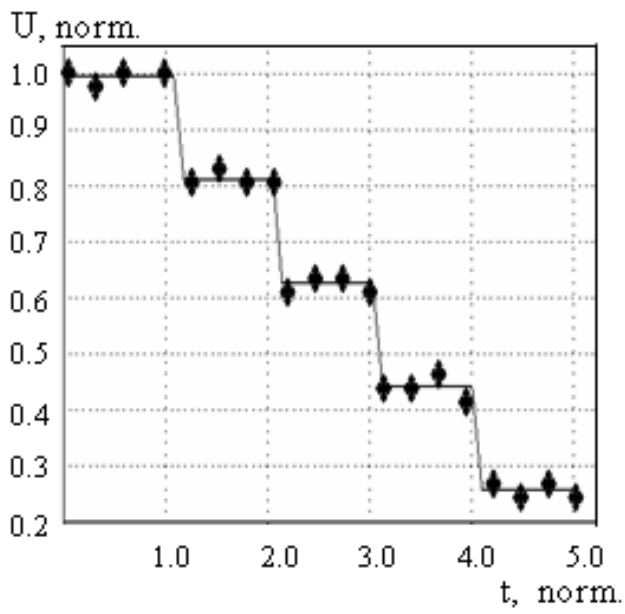

Figure 6. The temporal response of a sensor control. By tags the experimental points are shown

Identical portions of ammonia it was entered into a container cross identical time slots (approximately $2.5 \mathrm{~s}$ ). As a result it is possible to draw following conclusions. i). Mean value of amplitude of the response on any section of detection differs from mean value of amplitude on adjacent sections approximately on identical value, and difference makes less than 5\%. ii) The arithmetic mean deviation of amplitude of the response from mean value during any period of detection is approximately equal to zero. iii) Measurement setup has a time response lowing one second.

The resulted experimental data testify to comprehensible metrological characteristics of the given chemical sensor control.

\section{Sensitivity Modification}

The decision of the task of increase of sensitivity WOCS can be carried out by means of the following construction of the instrument which is presented on Figure 7.

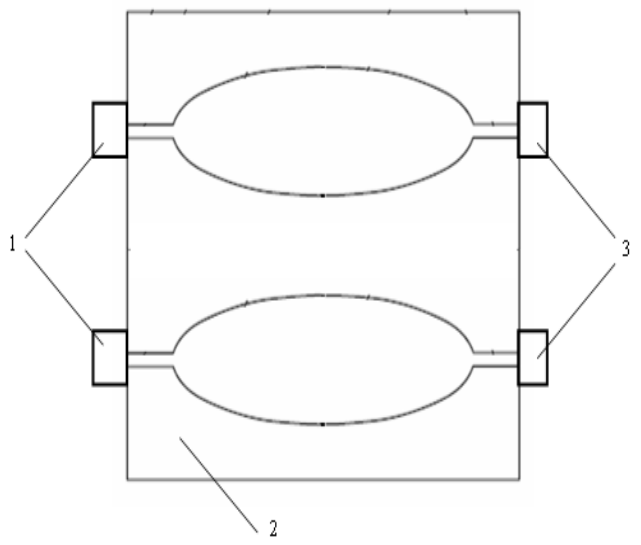

Figure 7. Schematic of the WOCS modification: 1- laser, 2 - waveguide structure, 3 - fotodetector 
The topology modified WOCS turns out by double multiplexing initial. Each of two coherent sources of optical radiation is joined to an appropriate input channel wave guide. The past through the waveguide structure optical radiation is registered by means of highly sensitive photo-sensor elements. The signal from them arrives in the computer where the generalised output signal WOCS is calculated.

Ammonia concentration is easily calculated using the Bouguer-Lambert-Beer law [1]

$$
P_{\text {out }}^{\prime}=P_{\text {out }} \exp \int_{-l / 2}^{l / 2} d x \sigma N(x)
$$

where $P_{\text {out }}$ is the radiation power at the output of the

waveguide cell at the absence of ammonia, $P_{\text {out }}^{\prime}$ is the output power in the presence of ammonia, and $l$ is the thickness of the layer of the medium, which corresponds to the length of the absorbing cell. The absorption cross-section of the ammonia sample is $\sigma$ and $N(x)$ is the measured concentration. For the He-Ne laser $\sigma_{H_{3}} \approx$ $3.810-16 \mathrm{~cm}[3,4]$. Rearranging Equation 4 then yields the ammonia concentration as

$$
N_{H N_{3}}=\frac{\ln \left(P_{\text {out }} / P_{\text {out }}^{\prime}\right)}{\sigma_{H N_{3}} L_{\text {eff }}}
$$

Using the experimental data obtained from the waveguide sensor shown in Figure 5, we were able to use (4) to determine an ammonia concentration of approximately 0.1 $\mathrm{mg} / \mathrm{m}^{3}$.

Similarly, the ammonia concentration measured at the limiting sensitivity of the double waveguide sensor can be obtained using (5):

$$
N_{H N_{3}}^{\min }=\frac{\ln \left(P_{\text {out }} / P_{\text {out }}^{\prime} \min ^{\prime}\right)}{2 \sigma_{H N_{3}} L_{\text {eff }}},
$$

where $P_{\text {out }}^{\prime}$ in is the noise level measured at the cell output in the absence of ammonia.

The theoretical estimation of limiting concentration of ammonia by which it is possible to measure by means of the considered device, received according to resulted in $[2,3]$ technique at known parameters of the task for $L_{\text {eff }}=62 \mathrm{~mm}$, $\mathrm{W}_{\max }=188 \mu \mathrm{m}$ and for a signal-to-noise ratio of about 20 makes $0.02 \mathrm{mg} / \mathrm{m}^{3}$.

\section{Conclusion}

The newly developed method enjoy the advantages of an optical channel for data transfer (parallel data processing in real time, high operating speed, and noise immunity) and the advantages typical of integrated optics (compactness, low power consumption, high sensitivity in information signal processing, stability against external action, and the possibility of using the batch-fabrication technique).

The developed devices under condition of precision measurements and their subsequent computer handling show excellent metrological characteristics. Reduction of optical losses and magnification of effective length - the main methods of improving of principal characteristics WOCS fulfilled on a Y-cut lithium niobate. Usage of such sort of sensors has good perspectives practically in all branches of a science and technique, especially there where telecommunication technologies are already used.

These sensors are also attractive for monitoring both gaseous pollutants and substances dissolved in liquids for a variety of biomedical, physicochemical, and environmental measurements. Of interest is also their potential integration in optoelectronic air quality control systems.

\section{Acknowledgements}

The author is grateful to M.Yu. Kvasha for creation of the experimental sample and support with measurements. The author is grateful to Prof. S.M. Zakharov and M.Yu. Kvasha for helpful discussion.

\section{REFERENCES}

[1] R. Klein, E. Voges. Integrated-optic ammonia sensor, Fresenius J. Analitical Chemistry, Vol. 349, No.4, 394-398, 1994.

[2] A. D'Orazio, M. De Sario, C. Giasi, L. Mescia, V. Petruzzelli, F. Prudenzano. Design of planar optic sensors for hydrocarbon detection. Optical and Quantum Electronics, Vol. 36, No. 6, 507-526, 2004.

[3] M. Bednorz, M. Urbańczyk, T. Pustelny, A. Piotrowska, E. Papis, Z. Sidor, E. Kamińska. Application of SU8 polymer in waveguide interferometer ammonia sensor, Molecular and Quantum Acoustics, Vol. 27, No.1, 31-40, 2006.

[4] A. A. Egorov, M. A. Egorov, Yu. I. Tsareva, T. K. Chekhlova. Study of the integrated-optical concentration sensor for gaseous substances, Laser Physics, Vol. 17, No.1, 50-53, 2007.

[5] E.N. Epikhin, M.Y. Kvasha, N.V. Masalsky, N.V. Praschikin, V.A. Volkov, K.-L. Paap. Computer simulation and experimental research of waveguide acoustooptical frequency modulators used for fiber optical gyroscopes, Photonics and Optoelectronics, Vol. 2, No.2, 137 - 148, 1994. 
[6] P.V. Lambeck, H.J.W.M. Hoekstra, J. van Lith, G. van Elzakker. Two novel integrated optical sensor types for measuring chemical concentrations, based on chemically induced changes of modal field profiles, J. of Nonlinear Optical Physics \& Materials, Vol. 13, No.2, 209-227, 2004.

[7] N. V. Masalsky. Theoretical research and computer simulation of waveguide sensors for pressure-measuring laser system, Laser Physics, Vol. 14, No.10, 1440-1443, 2004.

[8] R. G. Hunsperger. Integrated Optics: Theory and Technology, Springer, New York, 1991.

[9] J. Buus. The effective index method and its application to semiconductor laser, IEEE J. Quant. Elect., Vol. 18, No.9, 1083-1089, 1982. 